\title{
The influence of concentrative meditation training on the development of attention networks during early adolescence
}

\author{
Shruti Baijal' , Amishi P. Jha ${ }^{2}$, Anastasia Kiyonaga ${ }^{3}$, Richa Singh ${ }^{1}$ and Narayanan Srinivasan ${ }^{\text {* }}$ \\ Centre of Behavioural and Cognitive Sciences, University of Allahabad, Allahabad, India \\ 2 Department of Psychology, University of Miami, Coral Gables, FL, USA \\ ${ }^{3}$ Department of Psychology, University of Pennsylvania, Philadelphia, PA, USA
}

\section{Edited by:}

Jessica S. Horst, University of Sussex,

UK

\section{Reviewed by:}

Vanessa R. Simmering, University of Wisconsin Madison, USA

Anna V. Fisher, Carnegie Mellon

University, USA

*Correspondence:

Narayanan Srinivasan, Centre of Behavioural and Cognitive Sciences,

University of Allahabad, Allahabad

211002, India.

e-mail:nsrini@cbcs.ac.in
We investigate if concentrative meditation training (CMT) offered during adolescent development benefits subsystems of attention using a quasi-experimental design. Attentional alerting, orienting, and conflict monitoring were examined using the Attention Network Test (ANT) in 13-15 year old children who received CMT as part of their school curriculum (CMT group: $N=79$ ) vs. those who received no such training (control group: $N=76$ ). Alerting and conflict monitoring, but not orienting, differed between the CMT and control group. Only conflict monitoring demonstrated age-related improvements, with smaller conflict effect scores in older vs. younger participants. The influence of CMT on this system was similar to the influence of developmental maturity, with smaller conflict effects in the CMT vs. control group. To examine if CMT might also bolster conflict-triggered upregulation of attentional control, conflict effects were evaluated as a function of previous trial conflict demands (high conflict vs. low conflict). Smaller current-trial conflict effects were observed when previous conflict was high vs. low, suggesting that similar to adults, when previous conflict was high (vs. low) children in this agerange proactively upregulated control so that subsequent trial performance was benefitted. The magnitude of conflict-triggered control upregulation was not bolstered by CMT but CMT did have an effect for current incongruent trials preceded by congruent trials. Thus, CMT's influence on attention may be tractable and specific; it may bolster attentional alerting, conflict monitoring and reactive control, but does not appear to improve orienting.

Keywords: meditation, development, attention, conflict monitoring, conflict adaptation

\section{INTRODUCTION}

Attention is a cognitive system essential for many forms of learning, as well as for regulating one's thoughts, emotions, and actions. This system can be fractionated into the three subsystems of alerting, orienting, and conflict monitoring (Posner and Petersen, 1990). Alerting involves achieving and maintaining a vigilant or alert state of preparedness for fight or flight decisions. Orienting restricts processing to the subset of inputs that are relevant for the current task goals. Conflict monitoring resolves conflict between goals and performance, and prioritizes among competing tasks (for review, see Fan et al., 2002). There is growing evidence of heterochronicity in the development of these subsystems from childhood into adulthood (see Rueda et al., 2005).

Alerting is thought to be fully mature by the age of 4 years (Rueda et al., 2004). While simple orienting (such as moving one's eyes to an external flash) appears to mature quite early as well, more complex orienting processes which involve selection of relevant information in the face of distraction, mature later and are not at adult-levels even by age nine (Huang-Pollock et al., 2007). Some studies on the development of orienting indicate very early development occurring before the age of six and very little improvement after that age (Trick and Enns, 1998). Conflict monitoring is protracted in its development and is not fully developed until age 25 (See Diamond, 2006 for review; but see Rueda et al., 2005). These developmental differences have been attributed to differing system-specific reliance on the prefrontal cortex, whose structural and morphological maturity is delayed until young adulthood (Huttenlocher and Dabholkar, 1997).

In addition to differences in their growth trajectories, the three subsystems of attention vary in their centrality to successful academic achievement. There is growing evidence that conflict monitoring is most important in the acquisition of literacy (McCandliss et al., 2003), numeracy, and in a wide variety of other skills (Posner and Rothbart, 2007). Of the three networks, only conflict monitoring has been positively associated with school math achievement and tests of IQ, and negatively associated with peer reports of anti-social behavior (e.g., Konrad et al., 2005; Checa et al., 2008). Thus, while all three systems dynamically interact to support complex behavior, conflict monitoring seems to be more influential than orienting and alerting for achievement in educational settings.

Research regarding the central importance of conflict monitoring in academic achievement has spurred the creation of programs specifically aiming to expedite its development. There are several recent examples of social-behavioral (Diamond et al., 2007) and computer-based programs used in young children (Rueda et al., 2005; Thorell et al., 2008; Rabiner et al., 2010) that report positive results. Yet, in their evaluation of program efficacy, none of these prior studies has examined the dynamic conflict-sensitive upregulation of control that is central to theories of conflict monitoring. 
In tasks involving conflict monitoring, conflict trials contain distracting stimulus features associated with an incorrect response concomitant with a task-relevant stimulus. Cognitive demands are higher, response times are slower, and accuracy is lower for conflict vs. no conflict trials because these distracting features trigger stimulus-response mappings that must be overcome for successful performance. The difference between high and low current-trial conflict scores, referred to as the "conflict effect," is smaller in older vs. younger children, and smaller in higher vs. lower achieving students (Fan et al., 2003; Rueda et al., 2004).

Computational theories of control processes like conflict monitoring and conflict adaptation that emphasize dynamic adjustments in control during response conflict tasks, have successfully demonstrated when control is most likely to be engaged, modulated, or withdrawn (Botvinick et al., 2001; Brown and Braver, 2008). In the adult literature on conflict monitoring, many studies have shown that the conflict effect score is modulated by the conflict demands on the previous trial. Specifically, performance on high-conflict trials is better following high (vs. low) conflict trials (Gratton et al., 1992; Botvinick et al., 1999; Kerns et al., 2004). This facilitated performance as a function of previous high conflict is referred to as conflict adaptation, and is proposed to result from a conflicttriggered upregulation in cognitive control. Post-conflict trials, thus, enjoy greater access to control resources to resolve interference from irrelevant stimulus features (Botvinick et al., 2001). This upregulation in control is proposed to rely upon the prefrontal cortex (see Mansouri et al., 2009). It is also referred to as a proactive, as opposed to reactive control process, since it entails biasing information-processing before the onset of a critical stimulus (see Boy et al., 2010).

While a great deal is known about proactive control processes such as working memory maintenance as function of development (see Diamond et al., 2007), academic achievement (see Gathercole et al., 2004), and mental training (Klingberg et al., 2005; Jha and Kiyonaga, 2010), very little is known about conflict adaptation's relationship to these contexts. No studies to date, have examined if conflict adaptation differs over the lifespan, academic achievement, or if it might be altered when children participate in programs designed to improve conflict monitoring.

Very few training programs for "augmented attention" in children have been rigorously tested for efficacy and most are still in beta-testing phases (see Rabiner et al., 2010). Investigating if and how conflict adaptation is altered with training may help uncover the mechanisms of action by which training programs are able to bolster attentional processes. If, for example, a training program improves current-trial conflict effect scores but only when previous trial conflict is high, there would be positive support for the hypothesis that training may be bolstering the ability to dynamically upregulate proactive cognitive control. This level of specificity in understanding the route by which training bolsters performance may help inform next-generation treatment and training programs. In addition, it may allow training to be better targeted to individuals' particular vulnerabilities. For example, training programs found to benefit proactive control might be offered to older adults (see Braver et al., 2007), patients with Parkinson's disease (See Bonnin et al., 2010), and incarcerated youth (Iselin and DeCoster, 2009) who all suffer from impairments in proactive but not reactive control.
One category of mental training techniques, which has been growing in popularity and in evidence-based support for salutary effects on attentional processes, is contemplative training involving meditation (see Baer et al., 2006). Two recent studies in pre-school and elementary-aged school children reported improvements in attentional processes after children participated in a multi-week meditation training (MT) program using mindfulness meditation (Napoli et al., 2005; Flook et al., 2010). Mindfulness is characterized by full attention to present-moment experience without judgment, elaboration, or emotional reactivity (see Jha et al., 2007). Mindfulness MT programs offer exercises and didactic guidance to help participants cultivate this mental mode. Numerous writings suggest that MT improves two disparate forms of attention described as "concentrative" and "receptive" also referred to as "open monitoring" attention (Brown, 1977; Delmonte, 1987; see also Lutz et al., 2008). In the former, attention is restricted to a specific focus, such as the breath or a phrase (mantra). In the latter, attention is instead "objectless" and the goal is simply to keep attention fully "readied" in the present moment of experience without orienting, directing, or limiting it in any way. Whereas extraneous stimuli are considered distractors in concentrative attention, in receptive attention no stimuli are extraneous because attention is open to the entire field of experience.

There are many striking parallels between traditional conceptualizations of concentrative and receptive attention in the context of meditation, and modern views of attention from cognitive neuroscience. Previous mindfulness studies in adults suggest that concentrative attention is similar to orienting and conflict monitoring, since both of these systems involve voluntary allocation of attention in the service of one's goals. In contrast, receptive attention is akin to keeping attention in a readied or alert state (see Jha et al., 2007). Many studies in adults have confirmed that MT alters specific subsystems of attention. MT emphasizing concentrative attention has been found to improve attentional orienting (Jha et al., 2007; van den Hurk et al., 2009) as well as conflict monitoring (Chan and Woollacott, 2007; Tang et al., 2007; van den Hurk et al., 2009), whereas MT emphasizing receptive attention has been found to improve attentional alerting (see Jha et al., 2007).

Yet other studies suggest that attentional changes may not be tied to the emphasis in training, but rather on the experience of the participants, with novices in meditation improving in concentrative attention and experts improving in receptive attention (for discussion see Jha et al., 2007). Thus, prior studies in meditation have reported changes in all three systems of attention, as well as many other aspects of attentional functioning in adults (BrefczynskiLewis et al., 2007; Slagter et al., 2007; Srinivasan and Baijal, 2007; Lutz et al., 2009; MacLean et al., 2010; for review see Lutz et al., 2008). In addition to training programs involving mindfulness meditation, training involving transcendental meditation has also found positive benefits in children and adults (Cranson et al., 1991; Rosaen and Benn, 2006). Transcendental meditation involves recitation and repetition of an individually prescribed phrase (mantra) and is thus, a form of concentrative meditation training (CMT) with many of reported salutary effects (see Wallace, 1970; Alexander et al., 1993; Vegors, 1999).

Since some studies with children (Napoli et al., 2005; Flook et al., 2010) and adults (Grossman et al., 2004) indicate possible benefits of MT, further investigation into its mechanisms of action 
is warranted and necessary. Yet prior studies of MT have primarily involved either young children or adults. Very little is known about the impact of MT in adolescents (but see Zyloska et al., 2008).

Herein, we aim to investigate if CMT influences multiple attentional processes including alerting, orienting, conflict monitoring, and conflict adaptation in adolescents. Monastic traditions, which offer CMT as part monastic training, do not offer formal meditation to young monks until early adolescence, coinciding with the age-range of our study population. Thus, from an Indo-Tibetan monastic perspective, that this age-range may offer a critical window in which learning these techniques can be beneficial. From a neurodevelopmental perspective, the adolescent brain may be in a critical period of functional development to benefit from CMT. The rate of PFC maturation in the form of pruning, as well as neural growth in the form of localized increases in callosal density near temporal-parietal regions reach peak levels in the 13-15 year agerange (see Thompson et al., 2000). Thus, engaging in mental training aimed at promoting greater attentional control might benefit from being offered in a critical window of developmental changes within brain structures known to subserve different attentional processes.

In the adult literature of meditation's impact on attentional processes, quasi-experimental studies on individuals who have long-term experience with meditation practices, and who have selfselected over their lifetimes, to engage in meditation practice, are far more common (e.g., Brefczynski-Lewis et al., 2007; Slagter et al., 2007; Lutz et al., 2009) than studies with randomized-controlled designs involving novices. The logic is that those with long-term practice, who have made a commitment to voluntarily engage in meditation practices, are likely to have performance benefits that are more stable, and thus more tractable and specific. Yet, these quasi-experimental studies suffer a significant weakness, precluding any causal claims. It is possible that the group-wise differences between those with MT vs. controls, could be due to selection biases, pre-existing differences, or motivational differences. Nonetheless, observational/quasi-experimental studies have been deemed a necessary pre-cursor to large-scale and costly randomized design studies to be conducted in novices.

The current study used a quasi-experimental design to examine if there were tractable effects of CMT on the three subsystems of attention and conflict adaptation in 13-15 year olds. Similar to quasi-experimental studies in adults, we did not randomly assign individuals to receive or engage in CMT. Instead, we examined if attending a school which offered CMT vs. a school that did not, would influence attentional functioning. The study was conducted in India, where there is normative cultural acceptance of attending a CMT school, and engaging in CMT, and the two schools participating in this study were approximately matched for socio-economic status of students.

\section{MATERIALS AND METHODS PARTICIPANTS}

Two groups of school-aged children were recruited from two schools in Allahabad. Both schools had similar demographics in terms of religion with majority of the students in both schools being Hindu. The meditating participants were recruited with prior consent from a public school where CMT (CMT group, $N=79$,
44 males) was provided as a part of their regular academic curriculum. The 13-year-old children in the CMT group $(N=29)$ had already received "relaxation training" (a pre-cursor to CMT) for at least one year (when they were 12-year-old) prior to participation in this study and in general, had spent a few months practicing meditation. The 14- $(N=26)$ and 15-year-old $(N=24)$ in the CMT group had received training in transcendental meditation for an average of at least 1 and 2 years respectively which involved daily exercises of $10 \mathrm{~min}$, twice a day, involving a silent mantra (sound) chanting as an object of meditation performed in a group setting at the school. Transcendental meditation is practiced worldwide by millions of people (for more details, see Shear, 2006). The training also included pulse reading for $1 \mathrm{~min}$ and pranayam (breathing exercise) for 2-3 min in the morning session.

The control participants (control group: $N=76,60$ males; $N=25$ in 13-year-old, $N=25$ in 14-year-old, and $N=26$ in 15-year-old) were recruited from a different public school, which did not provide CMT or any other form of MT. Both schools were comparable in terms of the socio-economic status and academic background of the students (if at all the school that provided MT is considered more likely to have students with lesser socio-economical status given that it was less expensive). Most of the students in the schools came from geographical area around the schools and informal interviews with teachers indicated that there was no special reason for most of the students attending the schools. The CMT school was less expensive to attend and not as well known as a specially desired school by self-report of the teachers in that school.

All students performed the Attention Network Test (ANT; Fan et al., 2002). Three children from the meditation group and five from the control group were excluded from data analysis for accuracy performance more than two SD below the group mean, leaving $N=76$ in the CMT group and $N=70$ in the control group for the final analysis. The study was approved by the University of Allahabad Institutional Ethics Review Board and informed consent/ ascent conformed to the University's ethical standard practices of research with children.

\section{STIMULI AND DESIGN}

All participants were given the ANT 1.3.0 version developed by Fan et al. (2002). Details about the stimulus conditions are given in Figure 1. Participants viewed a 15" laptop screen from a distance of $55 \mathrm{~cm}$ and responses were obtained through two input keys on the mouse. A central fixation cross was part of the standing background throughout the experiment. With the exception of no-cue trials, all trials began with the presentation of a warning cue for $100 \mathrm{~ms}$. Cue offset was followed by a $400 \mathrm{~ms}$ delay. Each trial ended with the presentation of a target that appeared $1.068^{\circ}$ above or below fixation. The target remained on the screen until a response was executed but for no longer than $1700 \mathrm{~ms}$. The inter-trial interval (ITI) varied randomly from 400 to $1600 \mathrm{~ms}$ across trials. One hundred ms with no stimuli were added to the ITI of no-cue trials so that the total trial duration for all trial types was comparable.

The target was an arrow pointing either leftward or rightward. The participant's task was to indicate the direction in which the arrow pointed, as quickly and accurately as possible, while maintaining fixation on the central cross throughout the trial. The participants responded with the index finger of each hand and were to 
press the left or right button on the mouse to indicate their response. In most cases, four arrows - two to the right and two to the left flanked the target arrow, and the participant's task was to indicate the direction of only the center arrow. Thus, the target could be unflanked (neutral target), pointing in the same direction as the flanking arrows (congruent) or in the opposite direction (incongruent) of the flanking arrows. Each target presentation was preceded by one of four cue conditions: (1) no-cue: the fixation point remained on the screen during the cue presentation period but no cue was presented; (2) double-cue: asterisks appeared simultaneously at target positions above and below fixation; (3) center-cue: a single asterisk appeared at fixation; (4) spatial-cue: a single asterisk appeared at the location of the upcoming target. The spatial cue was $100 \%$ predictive of the target position and was equally likely to occur above or below the fixation point. Thus, cue conditions varied in the temporal and spatial information they provided about the subsequent target presentation. No-cue trials provided neither temporal nor spatial warning - the target simply appeared - while double- and center-cue trials provided only temporal warning, and spatial-cue trials provided both temporal and spatial warning about the upcoming target. After an initial practice session, all participants performed a total of 312 experimental trials.

\section{RESULTS}

All analyses were performed on reaction time (RT; ms) scores for correct trials only, and accuracy (\% correct) scores. The efficiency of each attentional network (alerting, orienting, and conflict

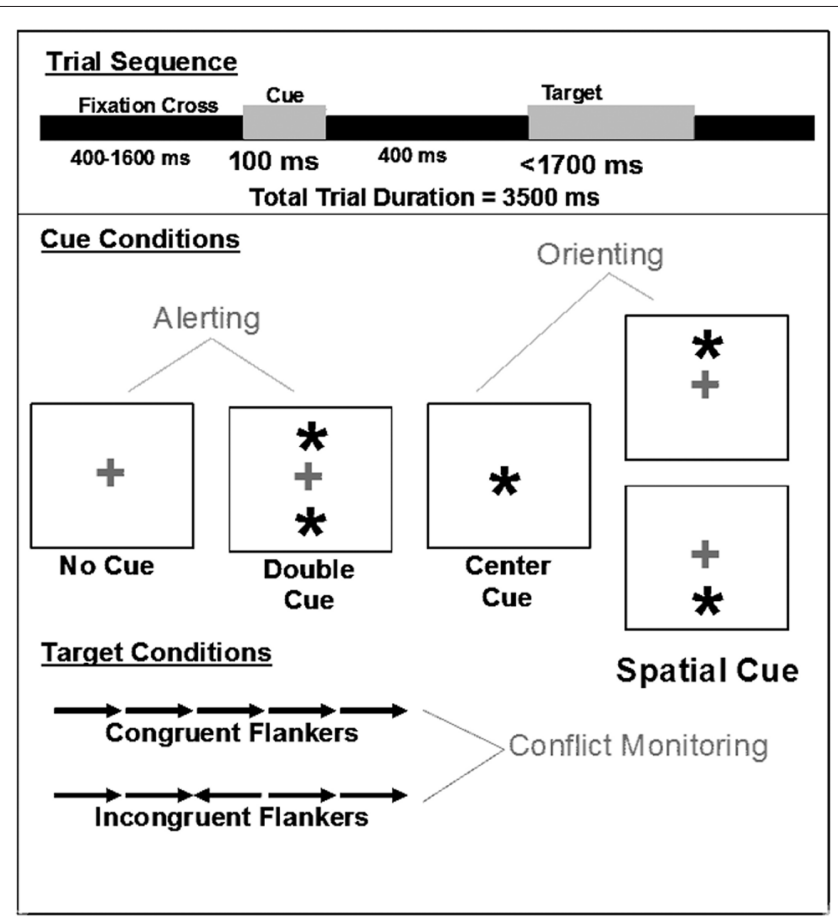

FIGURE 1 | Trial sequence and timing for the attention network test. Participants were instructed to keep their eyes fixated on the center cross during all trials. The experiment consisted of four cue conditions and two target conditions. All trial types were randomly intermixed and equally probable. monitoring) was examined separately via paired subtractions across subsets of conditions. These analyses considered only trials on which the target was flanked by arrows, excluding the neutral target condition. Alerting was indexed by the difference between scores on double-cue trials and no-cue trials (collapsed across target congruency condition). Orienting was indexed by the difference between scores on spatial-cue trials and center-cue trials (collapsed across target congruency). Conflict monitoring was indexed by the difference between scores on congruent target trials and those on incongruent target trials (collapsed across cue-type). The results of these paired subtractions will be referred to as subsystem difference scores. This method of analysis has been used extensively with the ANT and has been reported in detail elsewhere (e.g., Fan et al., 2002; Jha et al., 2007).

The mean subsystem difference scores for CMT and control participants of each age group are reported in Table 1. To explore the relationships between ANT subsystems, age, and MT in the academic context, we conducted univariate analyses of covariance (ANCOVA). Each analysis included a dependent variable of subsystem difference score (alerting, orienting, or conflict monitoring) for both RT and accuracy, the fixed factor of group (CMT vs. control) and the covariate of age (13-15 year olds).

The ANCOVAs for Alerting revealed no main effect of age, but did reveal a main effect of group for both $\mathrm{RT}[F(1,143)=4.22$, $p<0.05$; see Figure 2A] and accuracy $[F(1,143)=6.21, p=0.01]$. The Alerting subsystem difference scores were smaller for RTs and more positive for accuracy in the CMT vs. control group. The direction of these group-wise differences suggests that the CMT group had better alerting ability relative to the control group (see Fan et al., 2002). The ANCOVA for orienting revealed no main effects of age or group (see Figure 2B) for either RT or accuracy. The ANCOVA for conflict monitoring revealed a main effect of age for RT $[F(1,143)=5.86, p<0.05]$ and accuracy $[F(1,143)=8.37$, $p<0.01]$ and group (see Figure 2C) for both RT $[F(1,143)=5.51$, $p<0.05]$ and accuracy $[F(1,143)=4.01, p<0.05]$. Conflict monitoring scores were smaller in older vs. younger participants (Figure 3 ), and these scores were smaller in the CMT vs. control group, suggesting that greater developmental maturity and CMT both improve conflict monitoring ability. Analysis on RTs based on group and congruency indicated that the benefit due to CMT is obtained with incongruent trials $(p<0.05)$.

Table 1 | Mean subsystem difference scores for meditators and controls from each age group.

\begin{tabular}{|c|c|c|c|c|c|c|c|}
\hline \multirow[t]{2}{*}{ Group } & \multirow[t]{2}{*}{ Age } & \multicolumn{2}{|c|}{ Alerting } & \multicolumn{2}{|c|}{ Orienting } & \multicolumn{2}{|c|}{$\begin{array}{c}\text { Conflict } \\
\text { monitoring }\end{array}$} \\
\hline & & RT & $\begin{array}{l}\text { Accuracy } \\
(\%)\end{array}$ & RT & $\begin{array}{l}\text { Accuracy } \\
(\%)\end{array}$ & RT & $\begin{array}{l}\text { Accuracy } \\
(\%)\end{array}$ \\
\hline \multirow[t]{3}{*}{ Control } & 13 & 54.43 & -1.8 & 66.18 & 0.18 & 159.59 & 5.5 \\
\hline & 14 & 49.10 & -0.71 & 56.69 & 1.7 & 130.69 & 4.3 \\
\hline & 15 & 45.72 & -0.58 & 62.74 & 1.3 & 130.32 & 2.1 \\
\hline \multirow[t]{3}{*}{ Meditation } & 13 & 41.32 & -0.064 & 61.40 & 0.39 & 124.20 & 3.5 \\
\hline & 14 & 36.08 & 0.33 & 57.98 & 1.3 & 128.23 & 2.4 \\
\hline & 15 & 32.53 & 0.91 & 62.63 & 0.56 & 93.29 & 1.9 \\
\hline
\end{tabular}



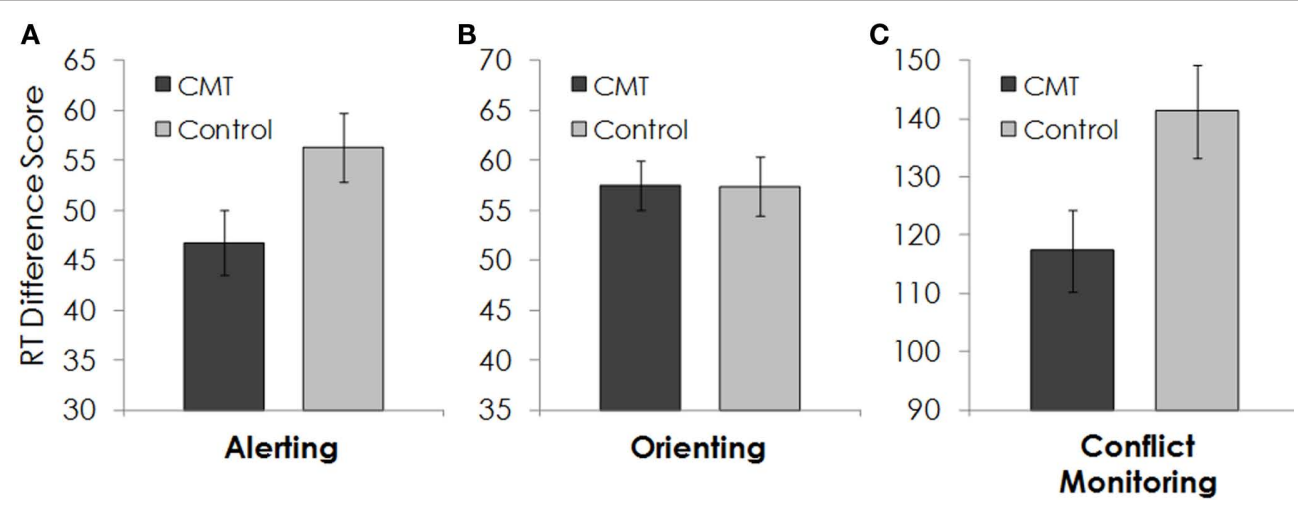

FIGURE 2 | Reaction time difference scores for (A) alerting, (B) orienting, and (C) conflict monitoring as a function of group.
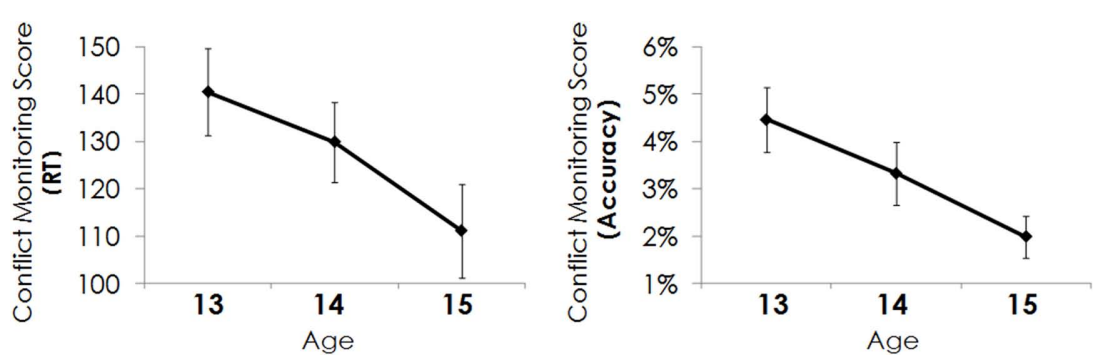

FIGURE 3 | (A) Reaction time conflict monitoring score for each age (collapsed across groups). (B) Accuracy conflict monitoring score for each age (collapsed across groups).

\section{CONFLICT ADAPTATION}

Based on prior conflict adaptation results, we investigated whether the target congruency condition of trial $n-1$ (the immediately preceding trial) might impact the magnitude of the conflict monitoring effect score on trial $n$ (the current trial), and whether the impact of trial $n-1$ congruency might also be sensitive to CMT. This sequential analysis as a function of previous trial type was conducted on only the conflict monitoring subsystem effect scores. The trial structure of the ANT precludes sequential trial-by-trial analysis of the trials comprising the other subsystems ${ }^{1}$.

To examine the influence of previous trial target congruency on conflict monitoring, we conducted repeated measures ANOVAs on conflict monitoring subsystem difference scores separately for RT and accuracy. The ANOVAs included the within subjects factor of previous congruency (target congruency on trial $n-1$; congruent vs. incongruent) and the between-subjects factor of group (CMT vs. control) and age (13 vs. 14 vs. 15). Previous congruent scores, for example, consisted of the magnitude of the conflict monitoring subsystem difference score calculated for only trials on which the preceding trial (of the current congruent or incongruent trial) had a congruent target. Likewise, previous incongruent difference

${ }^{1}$ While most trials have either an incongruent or congruent flanker condition in which the current trial was preceded by another flanker condition, the consecutive occurrence of trial types comprising the alerting and orienting systems are too rare to allow for a similar sequential analysis. scores were the conflict monitoring difference score only for current trials on which the preceding trial had an incongruent target. The results of these ANOVAs, for RT and accuracy conflict difference scores, are reported in Table 2.

There was a main effect of Previous Congruency for both RT, $F(1,140)=82.081, p<0.0001$ and accuracy $F(1,140)=32.960$, $p<0.001$; conflict monitoring scores were smaller when the previous trial had been an incongruent target. Importantly, there was no significant two way interaction between group and previous congruency for RT difference scores (See Figure 4). However, there was a close to significant interaction between group and previous congruency for accuracy difference scores, $F(1,140)=3.598, p=0.06$. While the conflict monitoring accuracy effect sizes were comparable across groups when the previous trial was incongruent, the CMT group had conflict monitoring scores of much smaller magnitude than the controls when the previous trial was a congruent target $(p<0.05)$. These results suggest that conflict monitoring was more efficient in the CMT vs. control group for previous congruent trials. There was a main effect of age for accuracy but not for RT. The interaction between age, group, and previous congruency was not significant for both accuracy and RT difference scores.

As reviewed above, the central analysis here for all three attention systems involved use of system-specific difference scores, as is typical in studies of the ANT (see Fan et al., 2002). Yet, for the conflict monitoring system, in particular, we wanted to ensure that performance on individual congruent and incongruent trials was in the expected 
direction, and that current $\times$ previous trial interactions were consistent with prior conflict adaptation results. Conflict adaptation studies do not typically use the difference score methodology, but rather an individual trial-specific analysis (see Egner, 2008). As such, ANOVAs were conducted on RTs and accuracy with training group and age as between-subjects factors, and two within subjects factors [currenttrial congruency (trial $n$ ): congruent vs. incongruent; and previous congruency (trial $n-1$ ): congruent vs. incongruent].

The results demonstrated (1) a significant main effect of current-trial congruency for RTs, $F(1,140)=533.754, p<0.0001$, and accuracy, $F(1,140)=80.771, p<0.001$, with faster RTs and higher accuracy for current congruent vs. incongruent trials; (2) a significant main effect of previous trial congruency for RTs, $F(1,140)=16.996, p<0.001$ and accuracy, $F(1,140)=, 30.728$, $p<0.001$, with faster and more accurate performance when the trial n-1 flankers were incongruent vs. congruent; and (3) a significant current congruency $\times$ previous congruency interactions for RTs, $F(1$, $140)=82.081, p<0.001$ and accuracy, $F(1,140)=32.960, p<0.001$.

Consistent with the conflict adaptation literature (See Egner, 2008) - for current congruent trials, participants were faster for previous congruent trials, but for current incongruent trials participants were significantly faster for previous incongruent trials $(p<0.05)$. Accuracy for current congruent trials was the comparable and near-ceiling regardless of the condition of the previous trial, but for current incongruent trials accuracy was higher for previous incongruent than previous congruent trials $(p<0.05)$. In terms of $\mathrm{CMT}$ and its effects on conflict adaptation, there was no three-way interaction for current congruency $\times$ previous congruency $\times$ group observed in the analysis of RTs (see Figure 4). However, the threeway current congruency $\times$ previous congruency $\times$ group interaction for accuracy was significant, which was driven by significant group-wise differences in only the trials that had current incongruent and previous congruent targets $(p<0.05)$, with higher accuracy scores in that condition for the CMT vs. control group (see Figure 5). Importantly, while there was a significant effect of age, the interaction between age, group, and previous congruency was not significant, nor was the four-way interaction.

Table 2 | Repeated measures ANOVA results for the conflict monitoring difference score, including the within-subjects effect of previous trial target congruency and between-subjects factor of group and age.

\begin{tabular}{|c|c|c|c|c|c|}
\hline \multirow[t]{2}{*}{ Effect } & \multicolumn{2}{|c|}{ RT } & \multicolumn{2}{|c|}{ Accuracy } & \multirow[t]{2}{*}{$d f$} \\
\hline & $\boldsymbol{F}$ & $p$ & $\boldsymbol{F}$ & $p$ & \\
\hline Group & 4.544 & 0.035 & 2.547 & 0.113 & 1,140 \\
\hline Age & 2.575 & 0.080 & 7.262 & 0.001 & 2,140 \\
\hline Group $\times$ age & 1.343 & 0.264 & 0.473 & 0.624 & 1,140 \\
\hline Previous congruency & 82.081 & 0.000 & 32.960 & 0.000 & 1,140 \\
\hline $\begin{array}{l}\text { Group } \times \text { previous } \\
\text { congruency }\end{array}$ & 0.321 & 0.572 & 3.598 & 0.060 & 1,140 \\
\hline $\begin{array}{l}\text { Age } \times \text { previous } \\
\text { congruency }\end{array}$ & 5.668 & 0.004 & 0.332 & 0.718 & 2,140 \\
\hline $\begin{array}{l}\text { Group } \times \text { age } \times \text { previous } \\
\text { congruency }\end{array}$ & 0.829 & 0.439 & 1.942 & 0.147 & 2,140 \\
\hline
\end{tabular}

Thus, these trial-specific analyses corroborate the findings reported for the conflict monitoring difference scores above. The results indicate that conflict adaptation is present in this age-range, with RT patterns comparable to those previously reported in adults (see Egner, 2008 for review). That is, 13-15 year old children are able to proactively upregulate control when previous trial was incongruent. The control adaptation RT patterns did not differ between the CMT and control group indicating that CMT might not produce a significant effect on conflict adaptation in this age-range, and may not improve proactive control.

The CMT-related improvements were not observed during task conditions in which proactive control was triggered by the task context - when the previous trial was incongruent vs. congruent; or when the need for attentional control was minimal - during current congruent trials. Instead, CMT's benefits were found in a task context devoid of proactive control triggers (previous congruent trial) but high current-trial demands for control to ensure correct performance (current incongruent trials).
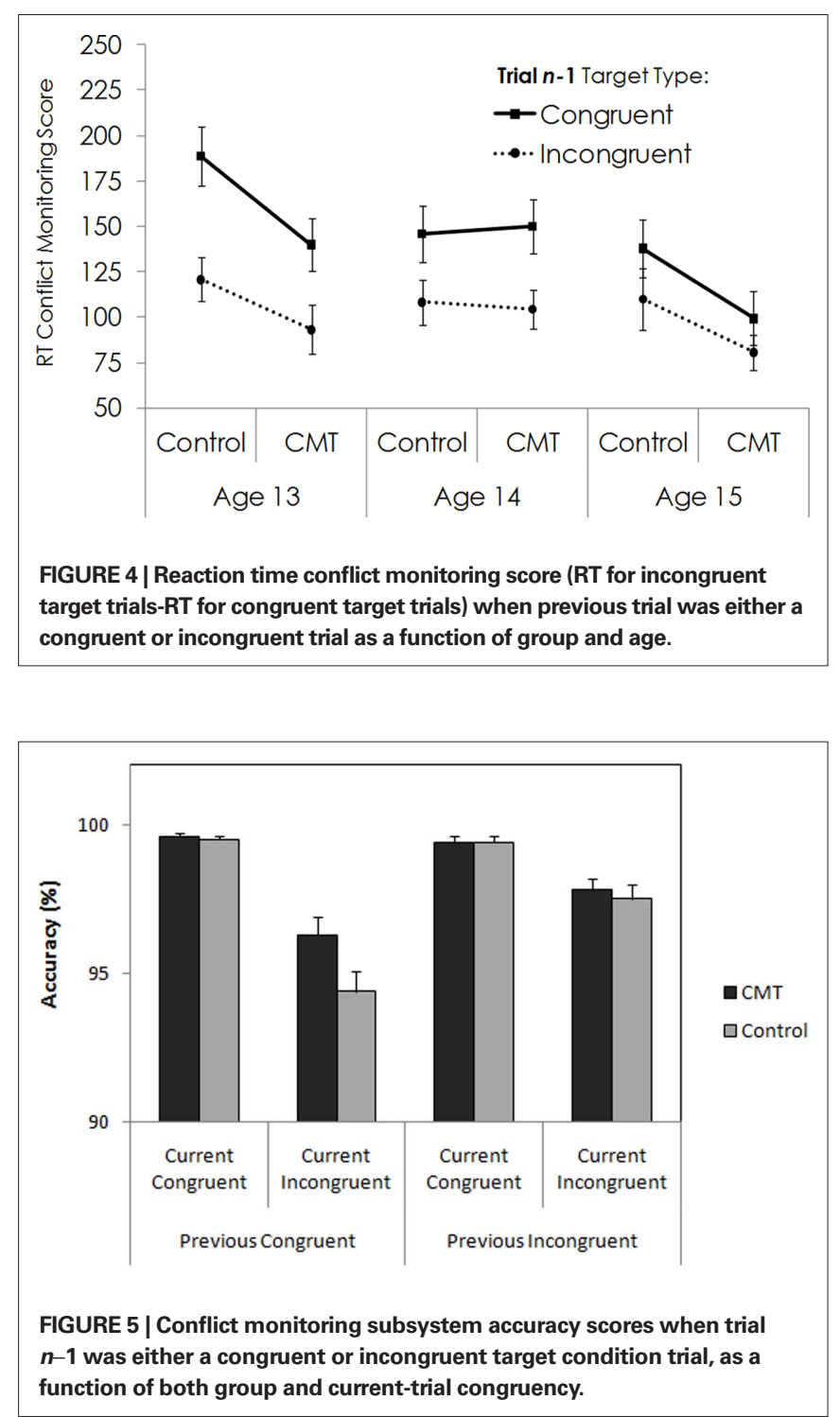


\section{DISCUSSION}

The current study aimed to examine the influence of long-term (1-3 years) practice of CMT, via enrollment in a school offering daily transcendental meditation exercises, on subsystems of attention in adolescents. Alerting and conflict monitoring, but not orienting differed between the CMT and control group. Conflict adaptation differed between the CMT and control group among the 13 year old children. Conflict monitoring demonstrated agerelated improvements, with smaller conflict effect scores in older vs. younger participants. The influence of membership in the CMT group on this system was similar to the influence of developmental maturity, with smaller conflict effects in the CMT vs. control group.

We acknowledge that the conclusions that can be drawn regarding the benefits of CMT on attention, are weak because of the quasiexperiment (vs. experimental) nature of our study design. Many factors other than group-wise differences of engaging in concentrative meditation practices could have contributed to the pattern of results observed herein. The students in the two training groups were not explicitly matched along genetic or demographic variables, such as parental/familial attitudes regarding meditation, meditation practice in the home, or baseline attentional or motivational differences. Thus, this study shares the pitfalls of many previous studies investigating the impact of meditation on attention, using similar designs, in adults (see Lutz et al., 2008). Nonetheless, attentional benefits of membership in the CMT group are consistent with the impact of CMT observed in previous studies in adults (see Jha et al., 2007). As such, while the nature of the design precludes causal claims regarding the beneficial effects of CMT, the results suggest that a full-scale randomized control trial in novices is warranted in this age-range.

Despite our inability to draw strong conclusion regarding CMT, this study does advance understanding of conflict adaptation effects in this age-range, which have not yet been reported in the developmental literature. All age groups demonstrated conflict adaptation in their RT scores, suggesting that children in the early adolescent age-range are capable of context-sensitive dynamic upregulation of proactive control. Yet there was no influence of CMT-group membership on the conflict adaptation RT effects. Although this is a null-result regarding training-group membership in conflict adaptation, we argue that this result does provide novel insight into the development of conflict adaptation, as well as its putative independence from systematic changes (that we observed to be tied to training-group membership) in conflict effect scores.

Counter to initial models on the topic (Botvinick et al., 2001), recent studies suggest that the nature of control operations supporting conflict resolution vs. conflict adaptation may be distinct (Bonnin et al., 2010; Boy et al., 2010). Conflict resolution occurs in response to experienced conflict when attentional control mechanisms have been used to overcome conflict after it has been experienced. Conflict adaptation refers to control mechanisms that are engaged prior to the appearance of the stimulus requiring conflict resolution. That is, whereas conflict resolution is reactive, conflict adaptation is considered a proactive control process (see Bonnin et al., 2010). As such, recent studies have argued (Boy et al., 2010) that the specific control operations engaged during conflict resolution and conflict adaptation may be distinct. Whereas conflict resolution involves engaging control to overcome the particular interfering, yet incorrect, motor programs activated by distracting flankers, for example, conflict adaptation may involve control processes which cannot be specific to the upcoming motor inhibition demands - since the upcoming trial has not yet occurred. Conflict adaptation may involve control processes that are able to be proactively prepared in the absence of specific stimulus features, such as narrowing or restricting of the aperture of attention so that only the narrow center of the target array is attended during flanker performance.

The current results are that conflict resolution, indexed by current-trial performance, regardless of previous trial conditions, was significantly improved in the students attending the CMT school vs. other school, in all age groups. While conflict adaptation was not improved in this group, relative to the control group in terms of RT, there was an improvement in terms of accuracy. This CMT-related benefit during previous trial analysis, involved a convergence of age and trial conditions in which proactive control was least likely to have been engaged. That is, the CMT group's accuracy was significantly better than the control group, when the current trial was incongruent and preceded by a congruent trial. This context may maximize the need to employ reactive control, and it was only under these circumstances that the CMT outperformed the control group.

Many questions remain for next-generation randomized control studies of CMT. For example, might CMT improve reactive control more so than proactive control? The ANT task used herein does not aim to fractionate attentional processes in these terms and is not well suited to answer this question. Studies using response conflict tasks in which the probability of congruent vs. incongruent trials is manipulated have been used previously to index the neural bases, and age-related changes in reactive vs. proactive control (see Braver et al., 2007). Future studies should examine the impact of CMT using this methodology. Nonetheless, it is noteworthy that similar to conflict adaptation, orienting, which has been considered by many models as an index of proactive control (see Shulman et al., 2001), was also insensitive to CMT. Yet, studies in novice and experienced adult MT-practitioners of mindfulness MT have found that orienting is bolstered with MT (see Jha et al., 2007; van den Hurk et al., 2009). Thus, it is not clear if the null effects in conflict adaptation and orienting observed herein are due to the specific nature of the training (CMT as opposed to mindfulness training) or the age of the participants (early adolescents as opposed to adults).

The current study found that in addition to conflict monitoring, alerting was bolstered by CMT group membership. Only one previous study has reported MT-related changes in the alerting system (Jha et al., 2007). Yet the alerting system benefits in the study of Jha et al. (2007) and those observed herein are quite distinct. Jha et al. (2007) reported improvements in the no-cue condition after participation in an intensive 1-month retreat in experienced adult MT-practitioners. These benefits were interpreted as improved readiness of attention, without provocation, due to the receptive skills cultivated during the 1-month retreat. In the current study, the direction of the RT effects observed in the CMT vs. control group in the calculation of the alerting difference scores, suggest that the CMT group was more responsive or "reactive" to the double-cue trials relative to the control group. Thus, the nature of the benefits of MT in the current study and that of Jha et al. (2007) appear to 
be distinct. Further, the pattern of results observed herein, are more in line with improvements in reactive control following CMT, more so than proactive control.

In addition to alerting, the current study found robust improvements in conflict monitoring. Prior studies have also reported this effect in not only the ANT task (Jha et al., 2007; Tang et al., 2007) but also Stroop interference tasks that bare similarity with our test of conflict monitoring, where interfering information needs to be inhibited (Wenk-Sormaz, 2005; Moore and Malinowski, 2009; but see Anderson et al., 2007). Neural evidence in adults also supports the hypothesis that MT may improve reactive control specifically in response to conflict (Baijal and Srinivasan, 2010).

It is also possible that the results herein are not due to CMT, per se, as previously discussed, or due to baseline cultural or genetic differences in children attending CMT schools, but are due to an "enrichment activity" culturally promoted to be beneficial in the school context. Might other types of enrichment activities, such as daily chess, art-class, yoga, dance, or other mental or physical training similarly improve specific aspects of attention more so than others? Future studies should compare CMT to an active comparison training group to address this limitation.

Many other next steps of research are warranted and motivated by the current findings. Given the special contribution of conflict monitoring with academic achievement (Rueda et al., 2005; Diamond et al., 2007; Thorell et al., 2008; Rabiner et al., 2010), it is interesting that this component was sensitive to age and CMT.

\section{REFERENCES}

Alexander, C. N., Swanson, G. C., Rainforth, M. V., Carlisle, T. W., Todd, C. C., and Oates, R. M. (1993). Effects of the transcendental meditation program on stress-reduction, health, and employee development: a prospective study in two occupational settings. Anxiety Stress Coping 6, 245-262.

Anderson, N. D., Lau, M. A., Segal, Z. V., and Bishop, S. R. (2007). Mindfulnessbased stress reduction and attentional control. Clin. Psychol. Psychother. 14, 449-463.

Baer, R. A., Smith, G. T., Hopkins, J., Krietemeyer, J., and Toney, L. (2006). Using self-report assessment methods to explore facets of mindfulness. Assessment 13, 27-45.

Baijal, S., and Srinivasan, N. (2010). Theta activity and meditative states: spectral changes during Sahaj Samadhi meditation. Cogn. Process. 11, 31-38.

Bonnin, C. A., Houeto, J., Gil, R., and Bouquet, C. A. (2010). Adjustments of conflict monitoring in Parkinson's disease. Neuropsychology 24, 542-546.

Botvinick, M., Nystrom, L. E., Fissell, K., Carter, C. S., and Cohen, J. D. (1999). Conflict monitoring versus selection-for-action in anterior cingulate cortex. Nature 402, 179-181.

Botvinick, M. M., Braver, T. S., Barch, D. M., Carter, C. S., and Cohen, J. D. (2001). Conflict monitoring and cognitive control. Psychol. Rev. 108 624-652.

Boy, F., Husain, M., and Sumner, P. (2010). Unconscious inhibition separates two forms of cognitive control. Proc. Natl. Acad. Sci. U.S.A. 107, 11134-11139.

Braver, T. S., Gray, J. R., and Burgess, G. C. of working memory variation: dual mechanisms of cognitive control," in Variation in Working Memory, eds A. Conway, C. Jarrold, M. Kane, A. Miyake, and J. Towse (Oxford: Oxford University Press), 76-106.

Brefczynski-Lewis, J.A., Lutz, A., Schaefer, H. S., Levinson, D. B., and Davidson, R. J. (2007). Neural correlates of attentional expertise in long-term meditation practitioners. Proc. Natl. Acad. Sci. U.S.A. 104, 11483-11488.

Brown, D. P. (1977). A model for the levels of concentrative meditation. Int. J. Clin. Exp. Hypn. 25, 236-273.

Brown, J. W., and Braver, T. S. (2008). A computational model of risk, conflict, and individual difference effects in the anterior cingulate cortex. Brain Res. 1202, 99-108.

Chan, D., and Woollacott, M. (2007). Effects of level of meditation experience on attentional focus: is the efficiency of executive or orientation networks improved? J. Altern. Complement. Med. 13, 651-657. (2007). "Explaining the many varieties

Future studies should examine if there is a correspondence between CMT and academic achievement. Also, since adolescence is an emotionally turbulent time with hormonal fluctuations and physical changes, future studies should investigate if and how CMT impacts emotion-regulation, self-esteem, and social functioning in schools. We appreciate that these types of studies may not be possible all over the world, and cultural acceptance of contemplative practices makes India a well-suited place to test this further. Yet, it is possible that the cultural climate itself mediates the benefits that are observed, which should be carefully considered in extrapolating from the current results.

Finally, the centrality of attention for academic achievement, protracted development of attention during childhood, and vulnerability of this cognitive system to environmental challenges all promote an enthusiasm for improving attention by various means - including contemplative training methods as investigated herein. No studies to date have examined the long-term consequences of such training methods on children as they enter adulthood. Longterm follow-up studies should be conducted to index the impact of training along multiple dimensions of functioning including conceptual learning, language learning, flexible, and creative thinking, and a host of other varieties of learning. Nonetheless, attentional training may be invaluable for children suffering from developmental disorders, such as ADHD, that have shown impairments particularly in the alerting and executive control networks of attention (Johnson et al., 2008) and may contribute to academic success.

Checa, P., Rodríguez-Bailón, R., and Rueda, M. R. (2008). Neurocognitive and temperamental systems of selfregulation and early adolescents' social and academic outcomes. Mind Brain Educ. 2, 177-187.

Cranson, R. W., Orme-Johnson, D. W. Gackenbach, J., Dillbeck, M. C., Jones, C. H., and Alexander, C. N. (1991). Transcendental meditation and improved performance on intelligence-related measures: a longitudinal study. Pers. Individ. Dif. 12, 1105-1116.

Delmonte, M. (1987). Constructivist view of meditation. Am. J. Psychother. 41, 286-298.

Diamond, A. (2006). "The early development of executive functions," in Lifespan Cognition: Mechanisms of Change, eds E. Bialystok and F. Craik (New York: Oxford University Press), 70-95.

Diamond, A., Barnett, W. S., Thomas, J., and Munro, S. (2007). Preschool program improves cognitive control. Science 318, 1387-1388.

Egner, T. (2008). Multiple conflictdriven control mechanisms in the human brain. Trends Cogn. Sci. 12, 374-380.

Fan, J., McCandliss, B. D., Sommer, T., Raz, M., and Posner, M. I. (2002). Testing the efficiency and independence of attentional networks. J. Cogn. Neurosci. 14, 340-347.
Fan, J., McCandliss, B. D., Thomas, K. M and Posner, M. I. (2003). Cognitive and brain consequences of conflict. Neuroimage 18, 42-57.

Flook, L., Smalley, S. L., Kitil, J. M., Galla, B. M., Kaiser-Greenland, S., Locke, J., Ishijima, E., and Kasari, C. (2010). Effects of mindful awareness practices on executive functions in elementary school children. J. Appl. Sch. Psychol. 26, 70-95.

Gathercole, S. E., Pickering, S. J., Knight, C., and Stegmann, Z. (2004). Working memory skills and educational attainment: Evidence from national curriculum assessments at 7 and 14 years of age. Appl. Cogn. Psychol. 18, 1-16.

Gratton, G., Coles, M. G., and Donchin, E. (1992). Optimizing the use of information: strategic control of activation of responses. J. Exp. Psychol. Gen. 121, 480-506.

Grossman, P., Niemann, L., Schmidt, S., and Walach, H. (2004). Mindfulnessbased stress reduction and health benefits. A meta-analysis. J. Psychosom. Res. 57, 35-43.

Huang-Pollock, C. L., Milkami, A. Y., Pfiffner, L., and McBurnett, K. (2007). ADHD subtype differences in motivational responsivity but not inhibitory control: evidence from a reward-based variation of the stop signal paradigm. $J$. Clin. Child Adol. Psychol. 36, 127-136. 
Huttenlocher, P. R., and Dabholkar, A. S. (1997). Regional differences in synaptogenesis in human cerebral cortex. J. Comp. Neurol. 387, 167-178.

Iselin, A. R., and DeCoster, J. (2009). Reactive and proactive control in incarcerated and community adolescents and young adults. Cogn. Dev. 24, 192-296.

Jha, A. P., and Kiyonaga, A. (2010). Working memory-triggered adjustments in dynamic control. J. Exp. Psychol. Learn. Mem. Cogn. 36, 1036-1042.

Jha, A. P., Krompinger, J., and Baime, M. J. (2007). Mindfulness training modifies subsystems of attention. Cogn. Affect. Behav. Neurosci. 7, 109-119.

Johnson, K. A., Robertson, I. H., Barry, E., Mulligan, A., Dábhis, A. Daly, M. Watchorn, A., Gill, M., and Bellgrove, M. A. (2008). Impaired conflict resolution and alerting in children with ADHD: evidence from the Attention Network Task (ANT). J. Child Psychol. Psychiatry 49, 1339-1347.

Kerns, J. G., Cohen, J. D., MacDonald, A. W. III, Cho, R. Y., Stenger, V. A., and Carter, C.S. (2004). Anterior cingulate conflict monitoring and adjustments in control. Science 303, 1023-1026.

Klingberg, T., Fernell, E., Olesen, P., Johnson,M.,Gustafsson, P.,Dahlström, K., Gillberg, C. G., Forssberg, H., and Westerberg, H. (2005). Computerized training of working memory in children with ADHD - a randomized, controlled trial. J. Am. Acad. Child Adol. Psychiatry 44, 177-186.

Konrad, K., Neufang, S., Thiel, C. M. Specht, K., Hanisch, C., Fan, J., Herpertz-Dahlmann, B., and Fink, G. R. (2005). Development of attentional networks: an fMRI study with children and adults. Neuroimage 28, 429-439.

Lutz, A., Slagter, H. A., Dunne, J. D., and Davidson, R. J. (2008). Attention regulation and monitoring in meditation. Trends Cogn. Sci. 12, 163-169.

Lutz, A., Slagter, H. A., Rawling, B. N., Francis, D. A., Greischar, L. L., and
Davidson, R. J. (2009). Mental training enhances stability of attention by reducing cortical noise. J. Neurosci. 29, 13418-13427.

MacLean, K. A., Ferrer, E., Aichele, S. R., Bridwell, D. A., Zanesco, A. P., Jacobs, T. L., King, B. G., Rosenberg, E. L., Sahdra, B. K., Shaver, P. R., Wallace, B. A., Mangun, G. R., and Saron, C. D. (2010). Intensive meditation training improves perceptual discrimination and sustained attention. Psychol. Sci. 21, 829-839.

Mansouri, F. A., Tanaka, K., and Buckley, M. J. (2009). Conflict-induced behavioural adjustment: a clue to the executive functions of the prefrontal cortex. Nat. Rev. Neurosci. 10, 141-152.

McCandliss, B. D., Beck, I., Sandak, R., and Perfetti, C. (2003). Focusing attention on decoding for children with poor reading skills: a study of the Word Building intervention. Sci. Stud. Read. 7, 75-105.

Moore, A., and Malinowski, P. (2009). Meditation, mindfulness and cognitive flexibility. Conscious. Cogn. 18, 176-186.

Napoli, M., Krech, P., and Holley, L. (2005). Mindfulness training for elementary school students: the attention academy program. J. Appl. Sch. Psychol. 21, 99-109.

Posner, M. I., and Petersen, S. E. (1990). The attention system of the human brain. Ann. Rev. Neurosci. 13, 25-42.

Posner, M. I., and Rothbart, M. K. (2007). Educating the Human Brain. Washington, DC: American Psychological Association.

Rabiner, D. L., Murray, D. W., Skinner, A. T., and Malone, P. S. (2010). A randomized trial of two promising computer-based interventions for students with attention difficulties. J. Abnorm. Child Psychol. 38, 131-142.

Rosaen, C., and Benn, R. (2006). The experience of transcendental meditation in middle school students: a qualitative report. Explore 2, 422-425.

Rueda, M. R., Fan, J., McCandliss, B. D., Halparin, J. D., Gruber, D. B.,
Lercari, L. P., and Posner, M. I. (2004). Development of attentional networks in childhood. Neuropsychologia 42, 1029-1040.

Rueda,M.R., Rothbart, M.K., McCandliss, B. D., Saccomanno, L., and Posner, M. I. (2005). Training, maturation, and genetic influences on the development of executive attention. Proc. Natl. Acad. Sci. U.S.A. 102, 14931-14936.

Shear, J. (2006). “Transcendental meditation," in The Experience of Meditation, ed. J. Shear (St. Paul, MN: Paragon House), 23-48.

Shulman, G. L., Ollinger, J. M. Linenweber, M., Petersen, S. E., and Corbetta, M. (2001). Multiple neural correlates of detection in the human brain. Proc. Natl. Acad. Sci. U.S.A. 98, 313-318.

Slagter, H. A., Lutz, A., Greischar, L. L., Francis, A. D., Nieuwenhuis, S., Davis, J. M., and Davidson, R. J. (2007). Mental training affects distribution of limited brain resources. PLoS Biol. 5: e318. doi:10.1371/journal. pbio. 0050138

Srinivasan, N., and Baijal, S. (2007). Concentrative meditation enhances pre-attentive processing. A mismatchnegativity study. Neuroreport 18 , 1709-1712.

Tang, Y., Ma, Y., Wang, J., Fan, Y., Feng, S., Lu, Q., Sui, D., Rothbart, M. K., and Posner, M. I. (2007). Short-term meditation training improves attention and self-regulation. Proc. Natl. Acad. Sci. U.S.A. 104, 17152-17156.

Thompson, P. M., Giedd, J. N., Woods, R. P., MacDonald, D., Evans, A. C., and Toga, A. W. (2000). Growth patterns in the developing brain detected by using continuum mechanical tensor maps. Nature 401, 190-193.

Thorell, L. B., Lindqvist, S., Bergman, S., Bohlin, G., and Klingberg, T. (2008). Training and transfer effects of excutive functions in preschool children. Dev. Sci. 11, 969-976.

Trick, L. M., and Enns, J. T. (1998). Lifespan changes in attention: the visual search task. Cogn. Dev. 13, 369-386. van den Hurk, P. A. M., Giommi, F. Gielen, S. C., Speckens, A. E. M., and Barendregt, H. P. (2009). Greater efficiency in attentional processing related to mindfulness meditation. Q. J. Exp. Psychol. 63, 1168-1180.

Vegors, S. (1999). Developing psychological health with transcendental meditation. Compl. Health. Prac. Rev. 5, 19-23.

Wallace, R. K. (1970). Physiological effects of Transcendental Meditation. Science 167, 1751-1754.

Wenk-Sormaz, H. (2005). Meditation can reduce habitual responding. Altern. Ther. Health. Med. 11, 42-58.

Zyloska, L., Ackerman, D. L., Yang, M., Futrell, J., Horton, N. L., Hale, T. S., Pataki, C., and Smalley, S. (2008). Mindfulness meditation training in adults and adolescents with attention deficit hyperactivity disorder - a feasibility study. J. Atten. Disord. 11, 737-746.

Conflict of Interest Statement: The authors declare that the research was conducted in the absence of any commercial or financial relationships that could be construed as a potential conflict of interest.

Received: 06 February 2011; accepted: 20 June 2011; published online: 12 July 2011.

Citation: Baijal S, Jha AP, Kiyonaga A, Singh R and Srinivasan N(2011) The influence of concentrative meditation training on the development of attention networks during early adolescence. Front. Psychology 2:153. doi: 10.3389/fpsyg.2011.00153 This article was submitted to Frontiers in Developmental Psychology, a specialty of Frontiers in Psychology.

Copyright (c) 2011 Baijal, Jha, Kiyonaga, Singh and Srinivasan. This is an open-access article subject to a non-exclusive license between the authors and Frontiers Media $S A$, which permits use, distribution and reproduction in other forums, provided the original authors and source are credited and other Frontiers conditions are complied with. 